\title{
Towards Realistic Mobility Models For Mobile Ad hoc Networks
}

\author{
Amit Jardosh, Elizabeth M. Belding-Royer, Kevin C. Almeroth, Subhash Suri \\ Department of Computer Science \\ University of California at Santa Barbara \\ Santa Barbara, CA - 93106 \\ \{amitj, ebelding, almeroth, suri\}@cs.ucsb.edu
}

\begin{abstract}
One of the most important methods for evaluating the characteristics of ad hoc networking protocols is through the use of simulation. Simulation provides researchers with a number of significant benefits, including repeatable scenarios, isolation of parameters, and exploration of a variety of metrics. The topology and movement of the nodes in the simulation are key factors in the performance of the network protocol under study. Once the nodes have been initially distributed, the mobility model dictates the movement of the nodes within the network. Because the mobility of the nodes directly impacts the performance of the protocols, simulation results obtained with unrealistic movement models may not correctly reflect the true performance of the protocols. The majority of existing mobility models for ad hoc networks do not provide realistic movement scenarios; they are limited to random walk models without any obstacles. In this paper, we propose to create more realistic movement models through the incorporation of obstacles. These obstacles are utilized to both restrict node movement as well as wireless transmissions. In addition to the inclusion of obstacles, we construct movement paths using the Voronoi diagram of obstacle vertices. Nodes can then be randomly distributed across the paths, and can use shortest path route computations to destinations at randomly chosen obstacles. Simulation results show that the use of obstacles and pathways has a significant impact on the performance of ad hoc network protocols.
\end{abstract}

\section{Categories and Subject Descriptors}

I.6.5 [Model Development] : Modeling methodologies

\section{General}

Performance

This work was supported in part by National Science Foundation grants EIA-0080134, IIS-0121562 and CCR-9901958.

Permission to make digital or hard copies of all or part of this work for personal or classroom use is granted without fee provided that copies are not made or distributed for profit or commercial advantage and that copies bear this notice and the full citation on the first page. To copy otherwise, to republish, to post on servers or to redistribute to lists, requires prior specific permission and/or a fee.

MobiCom'03, September 14-19, 2003, San Diego, California, USA.

Copyright 2003 ACM 1-58113-753-2/03/0009 ...\$5.00.

\section{Keywords}

Mobility models, Ad hoc Networks, Simulations

\section{INTRODUCTION}

The nature of mobile ad hoc networks makes simulation modeling an invaluable tool for understanding the operation of these networks. Wireless channels experience high variability in channel quality due to a variety of phenomenon, including multipath, fading, atmospheric effects, and obstacles. While real world tests are crucial for understanding the performance of mobile network protocols, simulation provides an environment with specific advantages over real world studies. These advantages include repeatable scenarios, isolation of parameters, and exploration of a variety of metrics. Repeatable scenarios aid in the development and refinement of networking protocols by allowing the protocol developer to make changes to the protocol and retest the protocol in the same scenario. This aids in deeper understanding of how the changes impact the performance results. Simulation also enables isolation of parameters. This allows the effects of a single parameter, such as mobility, data traffic or transmission range, to be studied in detail, while all other metrics are held constant. Additionally, simulation allows a wide variety of scenarios and network configurations to be evaluated. All of these characteristics are extremely difficult, if not impossible, with real world experiments. Due to these benefits, simulation has become a popular tool for the development and study of ad hoc networking protocols. The vast majority of networking protocols proposed for ad hoc networks have been evaluated with some simulation tool.

An important component of the network simulator is the mobility model. Once the nodes are initially placed, the mobility model dictates how the nodes move within the network. A variety of mobility models have been proposed for ad hoc networks [5, 7, 10, 12, 14, 19], and a survey of many is presented in $[2,6]$. These models vary widely in their movement characteristics. For instance, in the Random Walk mobility model described in [9], nodes select a direction in which to move, between 0 and $2 \pi$, a speed from a given distribution, and then move in that direction at that speed for either a specified number of steps or for a time period. At the end of this period, the nodes repeat this process. The Random Direction model [19] operates similarly to the Random Walk, except that nodes continue to walk until they are within some $\epsilon$ of the simulation boundary. Once they reach this area, they select a new direction in which to walk. 
One of the most popular mobility models for studying ad hoc networking protocols is the Random Waypoint model [5]. In this model, a node selects a random destination within the network, a speed from a distribution, and then moves to the selected destination at the selected speed. Once the node reaches the destination, the node rests for a pause time, and then repeats the process by selecting a new destination and speed and resuming movement.

What all of these models have in common is that the movement patterns they create are not necessarily comparable to true real world movement. In particular, people on college campuses, at conferences, and in shopping areas generally do not move in random directions. They tend to select a specific destination and follow a well-defined path to reach that destination. The selection of the path is influenced both by provided pathways and obstacles. For instance, on a college campus, individuals generally stay on paths that are provided for interconnecting the campus buildings. While certain individuals may stray from these paths (i.e., by cutting across lawns), the majority of people walk along the provided paths. Additionally, the destinations are typically not random, but are buildings, park benches, and other specific locations within the campus.

Previous research [6] has shown that the mobility model in use can significantly impact the performance of ad hoc routing protocols, including the packet delivery ratio, the control overhead, and the data packet delay. Hence, it is important to use mobility models that accurately represent the intended scenarios in which the protocol is likely to be utilized. In this way the performance of the protocol can be more accurately predicted.

In this paper, we propose to create more realistic movement models through the incorporation of obstacles, the construction of realistic movement paths, and the determination of signal-blocking regions created by the obstacles. The obstacles are placed within a network area to model the location of buildings within an environment, i.e., a college campus. Once the buildings are placed, we use the Voronoi diagram [15] of obstacle vertices to construct movement paths. Nodes are then randomly distributed across the paths. Destinations are selected from the set of obstacles, and shortest path route computations are used to determine the path each node will use to reach its selected destination. Finally, when nodes transmit, the obstacles obstruct the propagation of the transmission in an area defined as the obstruction cone of the node. Our specific contributions are the following:

- A mechanism for the placement of obstacles within a simulation terrain.

- The computation of pathways between the obstacles using Voronoi diagram computations.

- The calculation of the area in which the wireless signal is obstructed due to the obstacles.

- A mobility model that can easily be plugged into the GlomoSim network simulator [1] for use by other networking researchers.

To evaluate our mobility model, we use the Ad hoc OnDemand Distance Vector (AODV) routing protocol [17] and compare its performance with our model versus that utilizing the random waypoint model. Simulation results show that the use of obstacles and pathways have a significant impact on the performance of the AODV routing protocol.

The remainder of the paper is organized as follows. Section 2 details related research in the area of mobility modeling. Section 3 motivates the importance of creating realistic mobility models. Our proposed mobility model is described in detail in section 4 , while our modeling of transmission around obstacles is described in section 5. Section 6 presents the evaluation of our mobility model, and finally section 7 offers some concluding remarks.

\section{RELATED WORK}

There exists a wide variety of mobility models that have been postulated from both analytic and simulation-based studies on mobile systems. This section describes a sampling of these movement models that have been designed specifically for ad hoc networks. A concise categorization of mobility models can be found in [2], while a survey and simulation-based comparison of a variety of mobility models can be found in [6].

The mobility model described in [9] has become the foundation for a number of mobility models. In this model, each node selects a direction $\theta$ in which to travel from the range $[0 \ldots 2 \pi]$. The nodes select their speeds from a user-defined distribution of speeds, and then each node moves in its select direction at its selected speed. After some randomly chosen period of time, each node halts and selects a new direction and speed. It then resumes movement.

A number of variations of this model have been proposed. For instance, in the Random Direction model in [19], instead of moving for some period of time, each node moves until it reaches the boundary of the simulation area. It then selects a new direction in which to move. This model was created for the purpose of maintaining a constant density of nodes throughout the simulation. In $[11,16]$, a different variation of this model is used. When a node reaches the simulation area boundary, it is reflected back into the simulation area in the direction of either $-\theta$, if it is on a vertical edge, or $(\pi-\theta)$, if it is on a horizontal edge. The velocity of the node is held constant. Like these two models, the group mobility model presented in [12] is also based on the model in [9].

One of the most widely used mobility models is the Random Waypoint model [5]. In this model, each node selects a random point in the simulation area as its destination, and a speed $v$ from an input range $\left[v_{\min }, v_{\max }\right]$. The node then moves to its destination at its chosen speed. When the node reaches its destination, it rests for some pause time. At the end of this pause time, it selects a new destination and speed and resumes movement. The properties of the random waypoint model have been extensively studied [3, 4, 18, 19]. One of the interesting results of these studies addresses the node spatial distribution of the random waypoint model. It is shown that, due to the characteristics of the model, the concentration of nodes follows a cyclic pattern during the lifetime of the network. The nodes tend to congregate in the center of the simulation area, resulting in non-uniform network density.

One of the common characteristics of the previously described mobility models is that they all model the boundaries of the simulation area as a border that cannot be crossed. The Boundless Simulation Area Mobility Model described in [10] removes this limitation by allowing nodes to wrap around to the other side of the simulation area when 


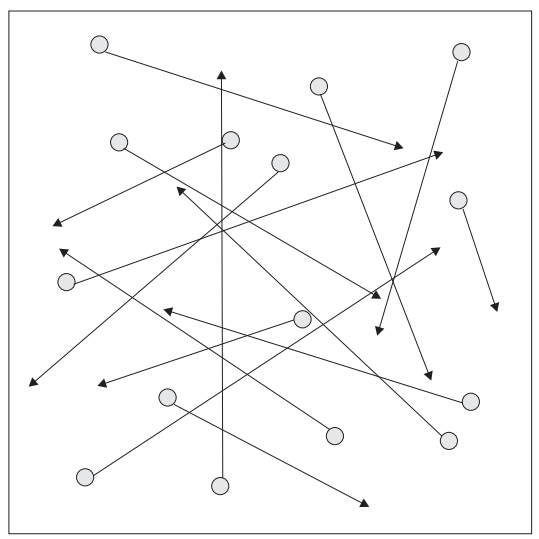

(a) Random Waypoint Mobility

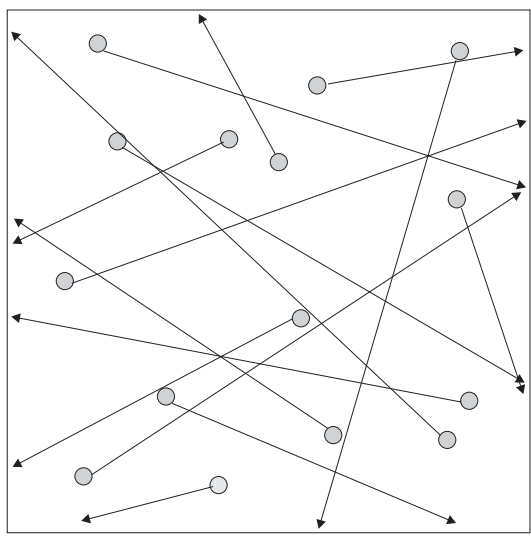

(b) Random Direction Mobility

Figure 1: Examples of Random Movement.

they encounter a border. The effect of this change is to create a simulation area modeled as a torus, rather than a rectangular surface.

While each of these models generates random mobility and can be used for the simulation study of ad hoc networking protocols, none of these models attempts to model the behavior of nodes in a realistic environment. The models assume open, unobstructed areas in which the nodes are free to move according to the constraints of the mobility model. In real-world scenarios, it is rare that groups of people are located in completely unobstructed areas; there are typically buildings, vegetation, benches, cars, and other objects that obstruct one's path. Additionally, it is unlikely to be the case that people follow random trajectories. On campuses people tend to follow provided pathways, in cities people follow sidewalks, in buildings people are confined to hallways, etc. While occasionally individuals may stray from the provided pathways, the majority of movement typically occurs along these paths. To understand how a protocol will perform in an obstructed environment, it is necessary to create mobility models that accurately model these environments. The Obstacle Mobility (OM) Model presented in this paper provides a mechanism for modeling movement in real world environments.

\section{MOTIVATION}

The mobility models described in section 2 have in common the characteristic of modeling movement in open, unobstructed areas. The nodes move randomly within these areas, stopping either at pre-selected destinations, bouncing off the network area walls, or moving through these walls to wrap around on the other side. While some models consider the previous velocity of the node when selecting its new velocity, other models select new movement directions without consideration of the node's movement history. In either case, the movement of the set of nodes is unlikely to represent true movement in the real world.

To illustrate this fact, figure 1 shows an example initial movement pattern of 15 nodes, randomly distributed in a simulation area. The movement pattern shown in fig- ure 1(a) represents movement in the random waypoint mobility model, while that shown in figure 1 (b) represents movement generated by the random direction model. As can be seen in the figure, nodes pick random destinations or directions within the simulations areas. The arrows represent the movement paths the nodes follow to their selected destinations. In the case of the random waypoint model, the figure illustrates the density waves phenomenon described in $[4,18,19]$, where nodes tend to cross through the center of the simulation area en route to their selected destinations. This creates a high concentration of nodes in the center of the network area until the nodes have moved sufficiently past this area. When this occurs, the density decreases once more. While the random direction model does not suffer from this problem, we argue that the movement generated by this model does not represent true movement any more accurately than the random waypoint model.

It has been demonstrated that the characteristics of the movement model significantly impact the resulting performance of ad hoc routing protocols [6]. Because of this result, it is exceedingly important to accurately represent the movement of mobile nodes so that the performance of the protocols under evaluation can be correctly depicted.

There are a variety of environments where the deployment of ad hoc networks is expected. A sampling of these include cities, campuses, highways, conferences and battlefields. What most of these environments have in common is the presence of obstacles that block node movement and that hinder propagation of wireless signals. Examples of obstacles include buildings, foliage, mountains, hillsides, cars, and people.

In this paper, we target scenarios that include the presence of buildings. These scenarios include college and business campuses, cities, and highways. We propose a mobility model that enables the placement of buildings of varying sizes. In this initial model, the obstacles are assumed to be completely obstructing; i.e., a radio transmission is completely blocked by the obstacle. In real scenarios, the quality of the transmission through a building is effected by the building's composition, as well as the thickness of its walls. 


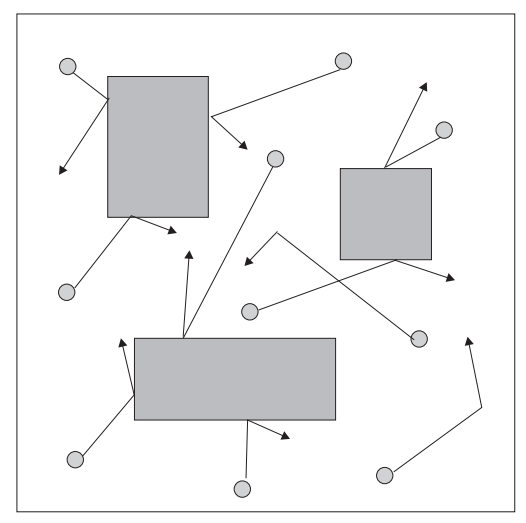

(a) Movement with Obstacles

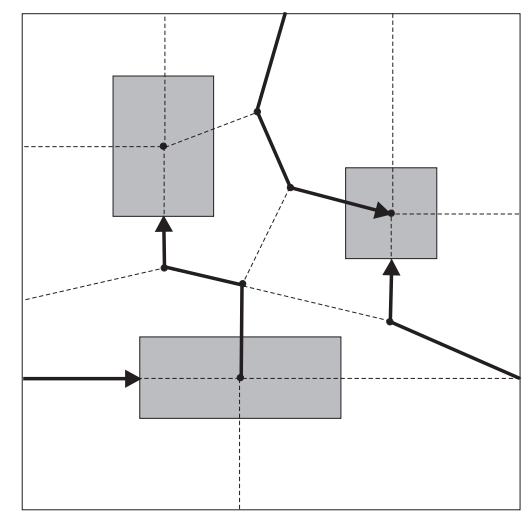

(b) Movement with Obstacles and Pre-Defined Pathways

Figure 2: Examples of Obstacle-based Movement.

Because modeling of these factors adds another layer of complexity to our model, we leave the inclusion of these factors for future work.

While the inclusion of obstructing objects is a step towards accurate modeling of realistic environments, it does not provide a complete solution. For instance, if the nodes were allowed to follow random paths within the network area, the movement patterns of the nodes would look similar to the example in figure 2(a). As is evident from the figure, what is lacking is movement paths for the nodes to traverse. In college campuses and city terrains, people do not randomly walk and reflect off of buildings; people follow pre-defined pathways (i.e., sidewalks) that interconnect buildings and lead into buildings. Typically, people select specific buildings, or other objects, such as benches or open grass, as their destinations. Hence, the inclusion of obstacles is not sufficient to create a realistic mobility model. Pathways connecting buildings must be computed to specify the movement paths of nodes.

After the location of the buildings has been determined, our mobility model computes pathways interconnecting and leading into and out of buildings using a Voronoi path computation [8]. The Voronoi path computation takes as input the coordinates of the buildings, and then computes pathways that define regions within the network area. These regions are created such that each point within a region has the same closest site (i.e., building). In other words, the pathways are equidistant from the buildings they lay between. An example of the Voronoi diagram for the topology given in figure $2(\mathrm{a})$ is shown in figure 2(b). While it may not be the case that pathways always lay equidistant between building cites (i.e., in a city, sidewalks would typically line one or both sides of the street), the calculation of these pathways creates pre-determined movement paths for the nodes to follow. Hence, it prevents the random movement illustrated in figure 2(a).

The following sections describe in detail the proposed mobility model and its effects on the movement and transmission ranges of the nodes within model.

\section{AN OBSTACLE MOBILITY MODEL}

Our proposed Obstacle Mobility (OM) Model has been designed to model the movement of mobile nodes in terrains that resemble real world topographies. The objects model buildings and other structures that provide a barrier to both the movement of the mobile nodes, as well as the wireless transmission of these nodes. In modeling such a terrain, a user can define the positions, shapes and sizes of these objects. Our model can handle arbitrary shapes and positions for the objects, allowing us to model many real-world terrains. The second component of our mobility model is a movement graph, which is a set of pathways along which the mobile nodes move. We use the Voronoi Diagram of the obstacle corners as our movement graph; this is a planar graph whose edges are line segments that are equidistant from two obstacle corners. Thus, the Voronoi diagram captures the intuition that pathways tend to lie "halfway in-between" adjacent buildings. Through the use of doorways on the sides of the building, we also allow movement through the buildings. The third and final component of the model is the route selection. We use the shortest path routing policy to move the nodes between two locations in the movement graph. That is, each node moves to its destination by following the shortest path in the Voronoi diagram, where the cost of each path segment is its Euclidean length.

Object locations and connecting pathways are computed once at the beginning of the simulation and do not change during the course of the simulation. The initial placement of the mobile nodes is obtained by distributing the nodes at random locations along the pathways. Each node selects a destination location, such as a building entrance, and then moves to that location using the shortest route from its current location. Thus, after the selection of a destination, each node runs a shortest path computation on the graph created by the pathways to determine the path it will traverse, and then moves towards that destination using its computed pathway.

Upon reaching its destination, the node pauses for some rest period. It then selects a new destination point, calculates the path it will take to reach the new destination, 


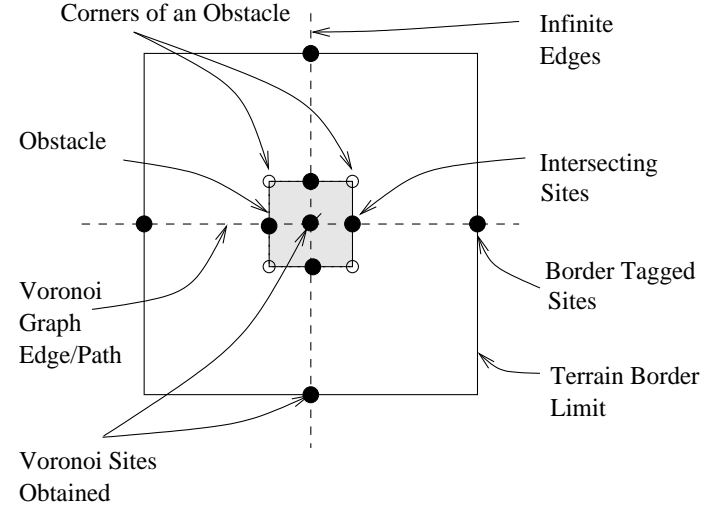

Figure 3: Sample Voronoi Diagram with Obstacle Coordinates and Sites.

and resumes movement. We point out that nodes can move through the buildings to reach their destinations; a shortest path between two locations can require going through a building, as is often the case in the real world.

The following sections describe in detail the construction and placement of obstacles, the Voronoi diagram computation, and the movement model.

\subsection{Obstacle Construction}

In our model, arbitrarily complex polygonal shapes can be used to specify the obstacles (buildings). Each polygonal shape is specified as an ordered sequence of its vertices (corners), where each vertex is defined by its coordinates. Non-linear shapes such as circles can be approximated by polygons, with the quality of approximation improving with the number of vertices in the polygon. In the simulations in section 6 , we were motivated to use a section of our university campus topography. Therefore, most of our examples use rectangular shaped obstacles. With rectangles alone, one can construct arbitrarily complex shapes by adjoining multiple rectangles. For instance, two adjoining rectangles form an $L$-shaped building, while three such rectangles can be used to form a $U$-shaped building.

Each side of the object (building) has one or more doorways through which the nodes can enter or leave the building. The shape and the placement of the obstacles has an effect on the node connectivity and mobility. In our model, we assume that obstacle walls are thick enough to completely block transmission of the wireless signal. We describe the modeling of transmission signals in section 5. Multipath fading of the signals, as well as shadowing effects are discussed in section 5.4 .

\subsection{Voronoi Graph and Pathways}

We now discuss how to model the potential pathways that exist in the presence of obstacles. There can be no single model that is the best for all terrains, but an appealing "geometry based" approach is to let the obstacles determine the pathways. Voronoi-diagram based pathways generalize the intuitive notion that the pathways typically run in the middle of the two adjacent buildings. Before we discuss our pathways, we briefly describe the classical notion of Voronoi diagrams from computational geometry.

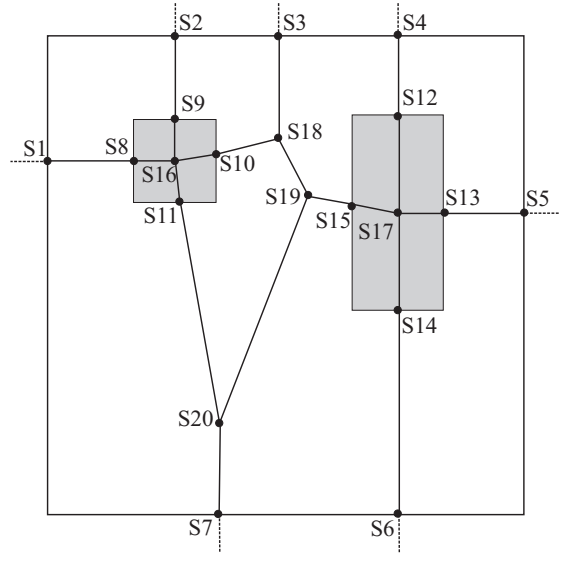

Figure 4: Example Terrain with Labeled Sites.

Consider a set of $n$ points $P=\left\{p_{1}, p_{2}, \ldots, p_{n}\right\}$ in the two-dimensional plane. For ease of reference, we call each of these points a location point. The Voronoi diagram of $P$ is a partition of the plane into convex polygonal cells, one cell per location point, so that every point in a cell is closer to its location point than to any other location point. Thus, a Voronoi cell of a location point $p_{i}$ can be thought of as $p_{i}$ 's region of influence. The boundary edges of the cells are straight line segments, and each segment is equidistant from its two closest location points. The Voronoi diagram of $n$ location points has $O(n)$ vertices and edges, and it can be computed in worst-case time $O(n \log n)[15]$.

The topological structure of a Voronoi diagram is an embedded planar graph with straight line edges. We will call this the Voronoi graph of $P$. Some of the edges of the diagram are semi-infinite ${ }^{1}$, and thus it is convenient to assume that the diagram is drawn on the surface of a sphere. In our case, we assume that the simulation is limited to a large square region of the plane, and so the Voronoi diagram is clipped inside the square, as shown in figure 3 . Thus, if our terrain had only point-size obstacles $P=\left\{p_{1}, p_{2}, \ldots, p_{n}\right\}$, then the Voronoi graph will represent a natural set of pathways; that is, the path segments lie at equal distance from the two closest obstacles (location points). We now describe how we build the Voronoi graph and pathways for our polygonal obstacles, and how we connect the pathways to buildings.

We use the corners of all the obstacles in our terrain as the set of location points. Thus, the example in figure 4 has eight location points, which are the corners of the two rectangular obstacles. The reader may note that the shown Voronoi diagram has eight cells (regions). The vertices of the Voronoi graph together with some additional vertices (defined below) act as the vertices of our pathways. First, we clip the Voronoi graph to lie entirely within the simulation region. The points of intersections between this outer boundary of the simulation region and the Voronoi graph also become the vertices in our pathways. Finally, the points of intersection between the Voronoi graph and the obstacles boundaries act as doorways.

\footnotetext{
${ }^{1} \mathrm{~A}$ semi-infinite edge is defined to be an edge that is unbounded on one end.
} 


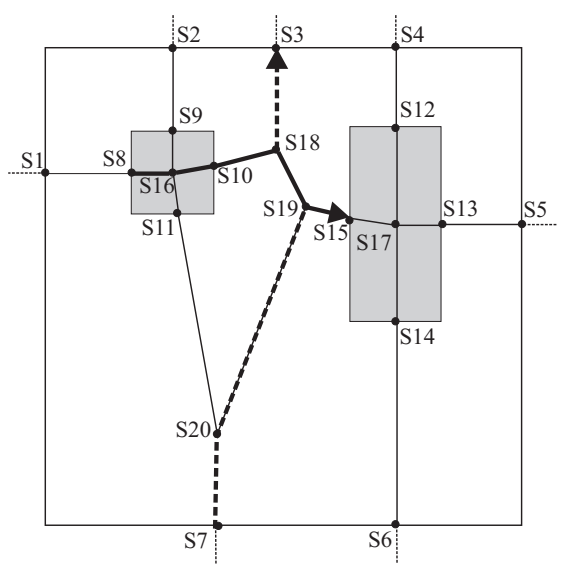

Figure 5: Example Movement Paths.

Figure 4 illustrates the computed Voronoi diagram for a network containing two objects. The set of sites is

$$
S=\left\{s_{1}, s_{2}, \ldots, s_{n}\right\} \cup\left\{s_{b 1}, s_{b 2}, \ldots, s_{b m}\right\} \cup\left\{s_{i 1}, s_{i 2}, \ldots, s_{i k}\right\},
$$

where $s_{1}$ to $s_{n}$ are the $n$ intersecting sites (e.g., $s_{8}$ through $s_{15}$ in figure 4$), s_{b 1}$ to $s_{b m}$ are the $m$ border sites (e.g., $s_{1}$ through $s_{7}$ ), and $s_{i 1}$ to $s_{i k}$ are the $k$ sites generated by the Voronoi computation (e.g., $s_{16}$ to $s_{20}$ ).

Given the set of sites $S$, the resulting set of edges generated by the Voronoi computation are

$$
E=\left\{e_{1}, e_{2}, \ldots, e_{l}\right\}
$$

where $e_{i}$ is an edge in the Voronoi diagram $D$.

\subsection{Semi-Definitive Node Movement}

In the proposed mobility model, nodes move along paths that are defined by the edges of the Voronoi diagram between the set of objects. These edges represent pathways that would typically be present connecting buildings on a business or college campus. Figure 5 represents examples of paths that may be selected in the given network. The random component of the movement is achieved through the initial placement of nodes at the sites within the network, and the selection of destination sites. Further, the speed at which nodes travel to their destinations, as well as the pause times once they reach their destinations, are randomly chosen from a distribution input by the user. Hence, different seed values can be used to create variations in the initial distribution of nodes, the selection of destinations, and the speed of movement.

Once the location of a node and its intended destination is determined, the path it will take to the destination is selected from the paths defined by the Voronoi diagram. The Voronoi diagram is pre-defined and establishes a path from each site to every other site. It is possible for the path between a pair of sites to traverse intermediate sites, as indicated by the example in figure 5 .

The Voronoi graph consists of undirected edges, where the weight of an edge is the length of that edge. Intuitively, a mobile user would tend to select the shortest path to its destination. Using this model, we can obtain the shortest path between a node's current location and its destination by running a shortest path computation, such as Dijkstra's algorithm. Once computed, the path to the destination site is maintained for each node. When the node reaches its destination, the process is repeated using the node's new location.

\section{TRANSMISSION BEHAVIOR}

The presence of objects in the network area influences the behavior of node transmissions. In our initial model, we assume that objects are substantial enough to prevent the passage of transmissions through their walls. We assume the use of omni-directional antennas by the mobile nodes. We model the behavior of the transmissions around the objects with the use of obstruction cones, as described in section 5.1.

In addition, we utilize a reachability matrix to represent the reception likelihood of a transmission between a pair of nodes. The use of this matrix is described in sections 5.2 and 5.3.

Propagation of signals, as well as fading and shadowing effects on the transmission are discussed in section 5.4.

\subsection{Obstruction Cones}

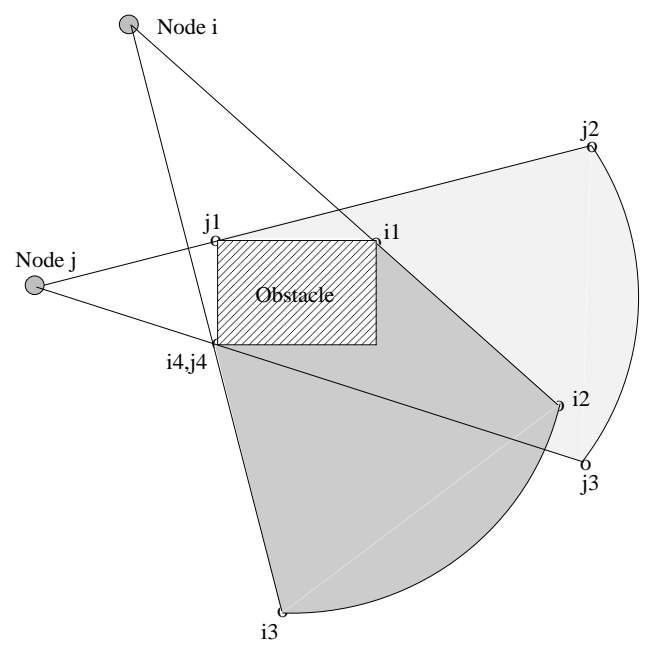

Figure 6: Obstruction Cones for Nodes $i$ and $j$.

The obstacles placed within the network have the effect of obstructing node transmissions. We assume the walls of the obstacles prevent the passage of signals between exterior and interior nodes. We model the propagation of signals around obstacles through the use of obstruction cones, as illustrated in figure 6 . Because there may be more than one obstacle in the omni-directional transmission range of a node, the node can have multiple obstruction cones. The obstruction set is then the set of all nodes located in the obstruction cones of a node. Obstruction sets can be represented as the following:

$$
\begin{aligned}
\text { OS }\left(\text { node }_{i}\right)= & \left\{\text { node }_{j} \mid j\right. \text { is not in the line of } \\
& \text { sight }(\text { LOS }) \text { of } i\}
\end{aligned}
$$

where node $_{j}$ represents a node $j$ that lies in the obstruction

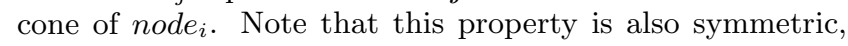
where

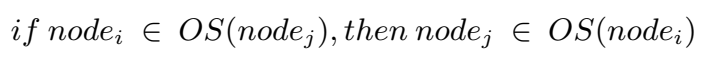


As figure 6 illustrates, transmission of the signal is completely blocked once the signal reaches the object; the signal does not pass through the object. For instance, node $i$ 's signal is blocked in the region defined by the points i1, $\mathrm{i} 2$, i3, and i4, while node $j$ 's signal is blocked in the region $\mathrm{j} 1, \mathrm{j} 2, \mathrm{j} 3$, and $\mathrm{j} 4$. In real-world scenarios, buildings and other obstacles are composed of different materials of varying thicknesses. The propagation of the signal through the obstacle is a function of both of these factors. In this initial model, we assume the obstacles block all signal propagation through their walls. Future modifications to our algorithm will consider the effects of non-opaque obstacles.

\subsection{Position Tags}

During the simulation, the position of each node is continually maintained. This assists the model in the quick computation of the obstruction cones. In addition to the coordinate location of the nodes, we introduce the notion of position tags for mobile nodes. Position tags indicate the location of the node with respect to whether it is currently exterior or interior to a simulation object. If the node is interior to an object, the tag indicates the object in which the node is located. Hence, each object is also given an identifier.

We define $t a g$ as the function that indicates the position properties of a node and $k$ as the integer identification given to an obstacle. Hence, we have

if $k \geq 1, \operatorname{tag}(i)=k$, else $\operatorname{tag}(i)=0$,

where $k$ represents the identifier of the obstacle in which the node is currently located, $k \in\{1 \ldots$ Number of objects $\}$ and $k=0$ denotes that the node is not currently located interior to an object.

\subsection{Reachability Matrix}

\begin{tabular}{|c|c|c|}
\hline & Noc & \\
\hline & Interior to an Object & Exterior to an Object \\
\hline 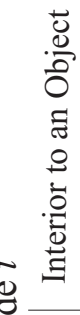 & $\begin{array}{l}\text { 0: if } i \text { and } j \text { lie within } \\
\text { disparate obstacles, or } \\
\text { there is no straight line } \\
\text { connecting } i \text { and } j \text { wholly } \\
\text { contained within the } \\
\text { object } \\
\text { 1: if } i \text { and } j \text { lie within the } \\
\text { same object and there is a } \\
\text { LOS path between } i \text { and } j\end{array}$ & 0 \\
\hline 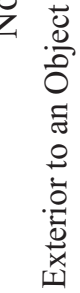 & 0 & $\begin{array}{l}\mathbf{0} \text { : if } i \text { is within the } \\
\text { Obstruction Cone of } j \\
\text { and there is not a LOS } \\
\text { path between } i \text { and } j \\
\text { 1: otherwise }\end{array}$ \\
\hline
\end{tabular}

Figure 7: Representation of Reachability Matrix.

To determine whether an object will influence the communication ability of a pair of nodes, we utilize a reachability matrix for the nodes. This matrix is treated as a reference matrix to represent the reachability of a transmission between a pair of nodes. The reachability matrix can be represented as shown in figure 7 . The rows and columns denote the placement of the transmitting and the receiving nodes, respectively. Hence, by the representation of the matrix, a reachability policy is determined. As shown in the figure, there are four possible cases for the node-pair configuration. An entry in the matrix for the two nodes $i$ and $j$ under consideration represents the reachability of a signal between the two nodes. A '1' indicates complete reachability, while a '0' indicates a completely blocked transmission. Further, if $N$ is the set of nodes, then $i, j \in N$. In the following notation, $I$ denotes the location of a node within an obstacle, while $E$ denotes the position of a node exterior to every obstacle in the network. The following discussion, as well as the reachability matrix, assumes the two nodes are within transmission range of each other.

- I-to-I : When two nodes are within the same obstacle and there is a straight line that can be drawn connecting the two nodes, the nodes are capable of communication with each other. In the case that two nodes are within the same obstacle and a straight line cannot be drawn that connects the two nodes, we assume that the two nodes cannot communicate. This can occur when nodes are located within concave polygons. In the case that the two nodes can communicate, the following property holds:

$\operatorname{tag}(i)=\operatorname{tag}(j)$ but $i \notin O S(j)$

- E-to-I : A transmission between nodes exterior and interior to an object is blocked because the nodes lie within the obstruction cone sets of each other.

- I-to-E : This scenario is the same as specified in the E-to-I case.

- E-to-E : This mode of transmission is dictated by the obstruction cone of the source node. The packets are dropped according to whether the destination is a member of the source node's obstruction set; i.e., if $j \in O S(i)$ then the transmission from node $i$ to node $j$ is blocked.

In our transmission model, when a node is attempting to transmit a packet to another node, the reachability matrix for that pair of nodes is checked. If the reachability matrix indicates that the packet should not be received by the receiver (due to, for instance, the receiver being located in the obstruction cone of the sender), the packet is dropped and prevented from reaching the receiver node. This action results in non-reception of the packet by the receiver node.

\subsection{Propagation Characteristics of Wireless Transmissions}

One of the primary limitations of the performance of wireless networks is the attenuation experienced by the signal as it propagates from the sender to the receiving node. In a setting with obstacles, the signal may reach the receiver via non-LOS (non-line-of-sight) propagation mechanisms, such as reflection, diffraction and scattering. This effect of multipath fading results in a drop in the Signal-to-Noise Ratio (SNR) of the received signal. The free-space fading models 
are not generally suited to calculate the attenuation undergone by the signal being received. Additionally, the fluctuations of the signal take place around a mean value and have long periods, leading to a phenomena called long-term fading that is characterized using lognormal distributions. In our simulations we have used the Two-Ray Pathloss Models that accommodate the reflections of the signals off the surface of the ground in addition to the direct path signals from the source transceiver to the destination transceiver.

Due to the factors of N-LOS propagation and lognormal fading (shadowing), in the proposed mobility model we assume that the signals received by the receiver are limited to direct paths only. We assume the average power of a received packet that is not received through LOS propagation is below the minimum SNR threshold.

As a consequence, when a signal is propagated between a pair of nodes and there is an obstacle obstructing the direct transmission path of the nodes, the signal is completely blocked by the obstacle. Thus the receiver fails to receive the transmission.

\section{SIMULATIONS}

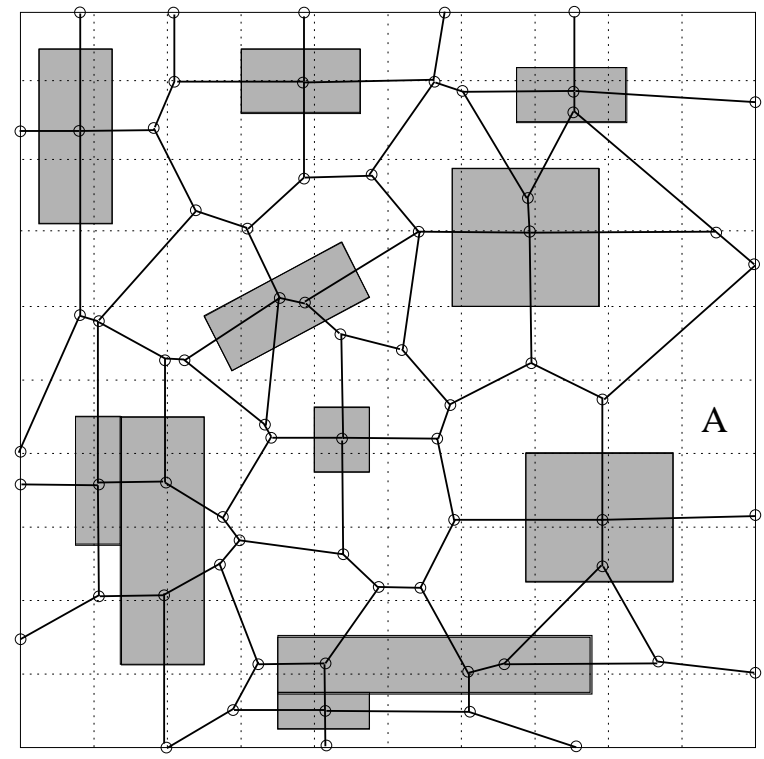

Figure 8: Simulated Terrain

The primary objective of our simulations is to understand the impact of obstacles in a simulation environment. To achieve this understanding, we evaluate two aspects of the Obstacle Mobility model. First, we determine the characteristics of the network topology created by this model. Due to the presence of obstacles and defined pathways, characteristics, such as the average node density, are likely to differ when compared with other mobility models. Second, we determine the impact of our mobility model on the performance of an ad hoc routing protocol.

Specifically, to understand the network topology characteristics create by our mobility model, we evaluate the following metrics:

- Node Density: Average number of neighbors per node.
- Path Length: Number of hops from a source to a destination.

For comparison, the above metrics are also evaluated for the random waypoint model.

To determine the impact of the obstacles and pathways on the performance of routing, we utilize the AODV protocol for route discovery and path set up. In these simulations, we also compare the results with the performance of AODV using the random waypoint model. The metrics we evaluate in these simulations are the following:

- Data Packet Reception: Number of data packets received at their intended destinations.

- Control Packet Overhead: Number of networklayer control packet transmissions.

- End-to-End Delay: End-to-end transmission time for data packets. This value includes delays due to route discovery.

The network scenario we utilize to evaluate the obstacleoriented mobility model is shown in figure 8 . This model was created based on the locations of buildings on the campus of the University of California at Santa Barbara. The model is a representation of the buildings on a selected area of the campus. We model an actual campus in order to create a realistic simulation terrain. The complexity of the geometric shapes of the buildings has been reduced by approximating the structures as units of rectangles, as described in section 4.1. The Voronoi paths are then generated based on this model. Interestingly, the Voronoi diagram generated by this model closely approximates the actual paths that exist around these buildings. At the beginning of simulations runs, the nodes are randomly placed at the sites composing the Voronoi graph.

When using the random waypoint model, there are no obstacles in the simulation area. At the beginning of the simulation, the nodes are randomly placed within the simulation area. The following sections give further details about the simulation parameters, as well as the obtained results. Simulated terrains, such as the one shown in figure 8, can be generated by a Java tool named TerGen, we developed for the project. The tool allows the user to specify the terrain size and place obstacles on the terrain. The tool then generates terrain files that are used as input by the network simulator to indicate the obstacles and compute the pathways.

\subsection{Simulation Environment}

All of the simulations were run using the GlomoSim network simulator [1]. The simulation area is $1000 \mathrm{~m} \times 1000 \mathrm{~m}$, and the maximum node transmission range is $250 \mathrm{~m}$. However, in the presence of obstructions, the actual transmission range of each individual node is likely to be limited. The propagation model is the two-ray pathloss model. At the MAC layer, the IEEE 802.11 DCF protocol is used, and the bandwidth is $2 \mathrm{Mbps}$. Because we are modeling a campus environment, the mobility of the nodes, unless otherwise stated, is randomly selected between 0 and $5 \mathrm{~m} / \mathrm{s}$ to represent walking speeds. The pause time in our simulations is also randomly selected between 10 and 300 seconds. Hence, when a node reaches its intended destination, it pauses for a certain period of time and then selects a new destination 


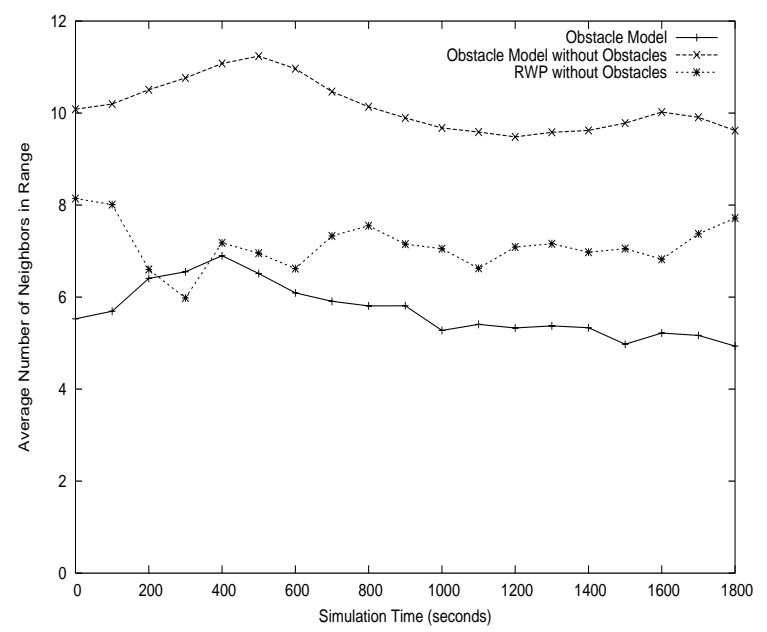

Figure 9: Node Density.

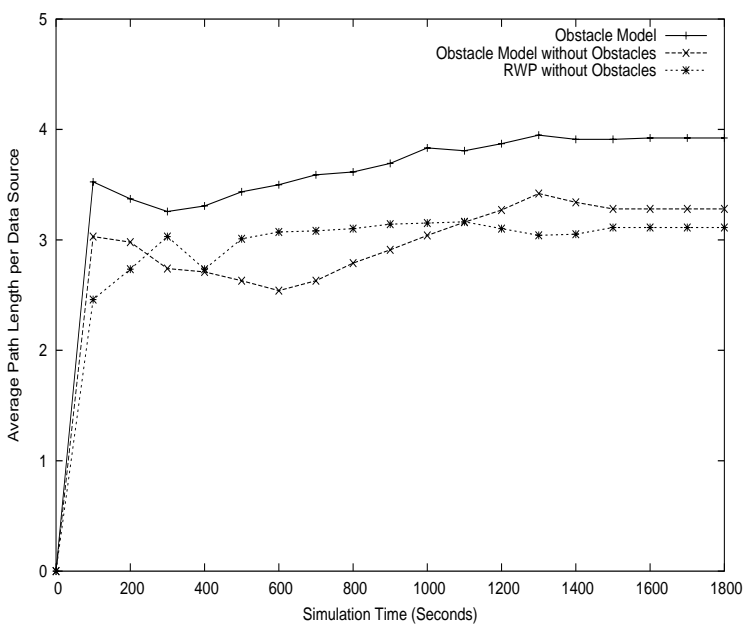

Figure 10: Path Length versus Time. and speed and continues movement. Each data point is an average of ten simulation runs with the nodes distributed in different initial positions.

To evaluate the characteristics of the network topologies created by the two mobility models, we randomly distribute the nodes at the beginning of the simulation. The number of neighbors per node is calculated for this initial distribution, as well as periodically throughout the simulation as the nodes move. The average path length is computed in two different scenarios. In the first, the nodes periodically discover routes to specified destinations throughout the simulation. The average discovered path length is plotted versus simulation time. In the second scenario, we vary the number of nodes between 20 and 100 . We allow the nodes to move for 60 seconds, at speeds between 0 and $5 \mathrm{~m} / \mathrm{s}$, so that they can distribute themselves randomly throughout the simulation area. Note that this is important for the obstacle mobility model since the initial placements for the nodes are only at the sites $S$ of the terrain, as described in section 4.2. 60 seconds after the start of each simulation run, ten route discoveries are performed, and the average discovered path length for each is recorded. The nodes continue movement while the route discoveries are taking place. The simulations run for a period of 1800 seconds.

For these scenarios, we study the impact of both the predefined movement pathways and the inclusion of obstacles. To perform experiments using only the pre-defined pathways, we utilize the obstacles to compute the pathways, and then remove them when computing neighbors and path lengths. When the obstacles are included, they are utilized to obstruct transmissions, as well as for the pathway calculations. In both cases, the objects are in the locations illustrated in figure 8.

The second set of simulations compares the performance of the AODV routing protocol using both the random waypoint and our obstacle mobility model. After the initial distribution of the nodes, the nodes move for 60 seconds so that they are distributed throughout the simulation area. Ten data sessions are then started. The data packet size is 512 bytes and the sending rate is 4 packets/second. The maximum number of packets that can be sent per data session is set to 6,000 . Hence, an aggregate of 60,000 packets can be received by the 10 destinations chosen. The ten sources and destinations are randomly selected. In these simulations, all nodes are assigned the same speed between 0 and $10 \mathrm{~m} / \mathrm{s}$, so that the effect of mobility can be determined. Movement continues throughout the simulations for a period of 1800 seconds.

\subsection{Results}

\section{Network Topology}

The average number of neighbors per node throughout the simulations is shown in figure 9. The number of neighbors per node for the random waypoint model matches those previously demonstrated for 50 nodes with $250 \mathrm{~m}$ transmission ranges in a $1000 \mathrm{~m} \times 1000 \mathrm{~m}$ area [13]. An interesting result is shown in this figure. Using the obstacle model with the obstacles, the average number of neighbors per node is considerably lower than in the random waypoint model. This can be explained by two reasons. The first is that nodes that are interior and exterior to obstacles are not able to communicate. Hence, nodes that are interior to obstacles only have as neighbors other nodes that are within line of sight in the same obstacle. This is likely to be a small number of nodes. Second, the obstacles block the propagation of the wireless transmissions of the nodes exterior to the obstacles. Hence, in many circumstances a node's neighbors are not all nodes within the omni-directional transmission range of the node; the transmission range is typically limited to some subset of this area. Hence, the number of neighbors is decreased.

For the mobility model with pathways but not obstacles, the nodes maintain their 360 degree omni-directional transmission ranges. In this scenario, the number of neighbors per node is much greater than in the other two mobility models. This is due to the limitation of where the nodes may travel. Because the nodes can only traverse the defined pathways, the area of the network that can be occupied by a node is reduced. The network area can be divided into a grid as shown in figure 8. In the random waypoint model, each grid box has an equal probability of containing one or more nodes. However, the obstacle model limits the number 


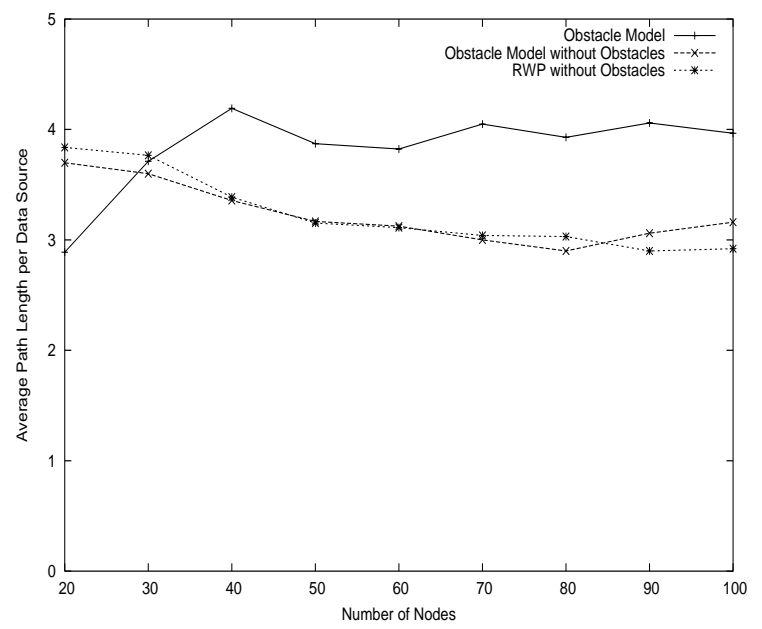

Figure 11: Path Length versus Number of Nodes.

of grid boxes that can contain mobile nodes to those containing pathways. For instance, a mobile node will never be located in the grid box labeled 'A' in figure 8 because there is no pathway contained in this area. Hence, by limiting the movement of the nodes to the pre-defined pathways, the effective area of the network in which nodes can be located is reduced. This leads to higher clustering of nodes, and hence a higher node density as indicated in figure 9 .

Figure 10 illustrates the average path length over time in each of the networks. The routes are initially discovered when the data sessions start. This occurs after 60 seconds of simulation time. The lengths of the paths are then tracked throughout the simulations. Path lengths may change due to link breaks and subsequent rediscovery of routes. The figure shows that the path lengths are roughly the same for the two mobility models in which obstacles do not block transmissions. The third mobility model shows an average $25 \%$ increase in the path length. In this scenario we can conclude that there is an average one-hop increase in the path length due to the presence of the obstacles. This increase is directly dependent on the topology of the network. For instance, if an obstacle was placed horizontally across $90 \%$ of the network width, the path length from one side of the obstacle to the other would be increased significantly.

What the figure does not represent, however, is the number of routes that were not able to be discovered due to sources and destinations being located interior and exterior to obstacles. To determine the effect of these obstructions, we measured the number of failed route discoveries for the ten data sessions. A failed route discovery is defined as the inability to discover a path between a source and destina-

\begin{tabular}{|c|c|c|}
\hline Mobility Model & $\begin{array}{c}\text { Initial Failed } \\
\text { Connections }\end{array}$ & $\begin{array}{c}\text { Total Failed } \\
\text { Connections }\end{array}$ \\
\hline \hline Random Waypoint & 0.21 & 1.73 \\
\hline Obstacle & 3.44 & 9.48 \\
\hline
\end{tabular}

Table 1: Number of Failed Connections.

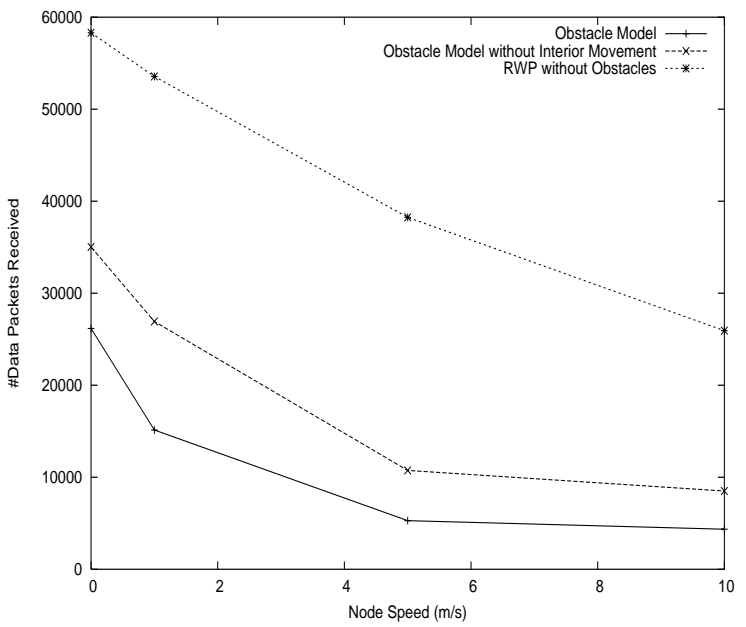

Figure 12: Data Packet Reception.

tion after the maximum number of RREQ attempts (three, in AODV). The results, averaged over ten simulation runs, are shown in table 1 . The table shows the number of failed route discoveries both for the initial route discovery for a destination and the total route discoveries during the simulation. Route discoveries can fail later in the simulation if a route breaks due to the source or destination moving into or out of an obstacle. As the table shows, the number of failed route discoveries is considerably higher in the network with obstacles than in the random waypoint model. This fact significantly impacts the routing performance results shown in the next section.

The average path length in networks with varying numbers of nodes is shown in figure 11. The figure shows that the path lengths are roughly the same for the mobility models in which obstacles do not block transmissions. The routes that are discovered involve nodes that are either randomly dispersed or on pre-defined pathways of the terrain considered, but, routes are not broken due to an obstruction. In the model that considers obstacles as an obstruction to transmission, the figure shows an increase in the average path lengths as the number of nodes within the network increase from 20 to 100 . When there are fewer nodes, the number of successful routes discovered is smaller due to the scarcity and dispersion of the nodes; there are fewer nodes to serve as relays for routes from a source to a destination. Hence, the probability of formation of long routes is low, and the routes that are successfully discovered are short. As the number of nodes increases, more nodes are available for route formation. For example, nodes on opposite sides of buildings can communicate since there are other nodes present to act as relays between the source and destination. Hence, on average, the number of successful route discoveries is greater, and these routes are longer than the routes discovered when there are fewer nodes.

\section{Routing Performance}

The total number of data packets received by their destinations is shown in figure 12 . The number of data packets received using the obstacle model is significantly lower than 


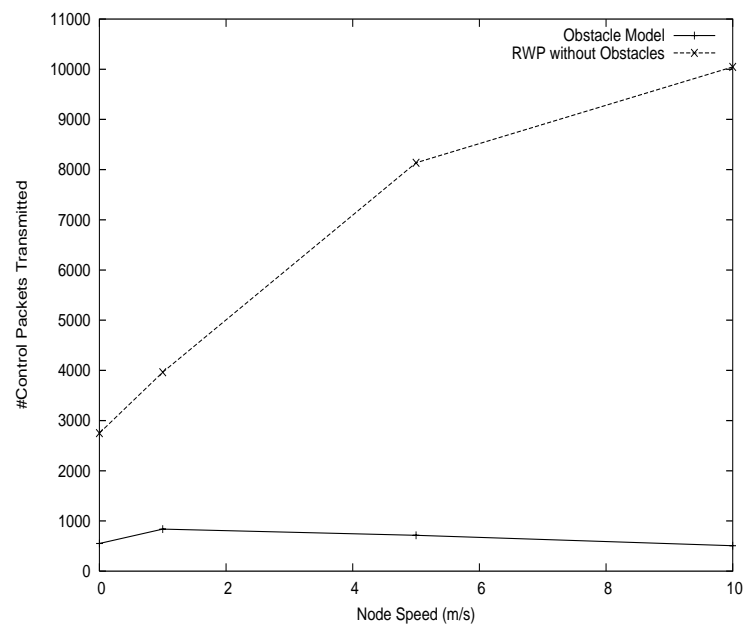

Figure 13: Control Packet Overhead.

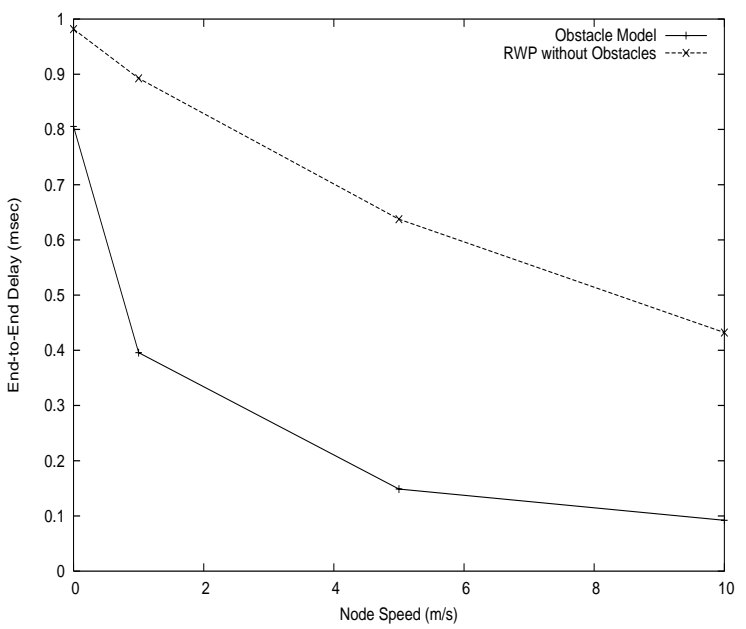

Figure 14: End-to-End Latency. that using the random waypoint model. This is due to the inability for routes to be discovered between sources that are interior(exterior) to an obstacle and destinations that are exterior(interior) to an obstacle. When the source and destination are either not both exterior to all obstacles or not both interior to the same obstacle, it is impossible for the two nodes to find a path to each other. As was shown in table 1 , the number of failed route discoveries with the obstacle mobility model is significantly higher than with the random waypoint. In our model, once the route discovery is deemed a failure, the data session between the source and destination is aborted; the route discovery is not re-attempted later in the simulation.

To isolate the impact of the obstacles on packet delivery, we evaluate the scenario where nodes are prevented from entering buildings. The nodes move only on paths on the exterior to the buildings. If the chosen path of a node should pass through the interior of a building, we prevent the interior movement and instead have the node 'jump' to the appropriate exit doorway. Although this movement is not realistic, it allows us to study the impact of the obstacles in obstructing transmissions. The data reception for this scenario is also shown in figure 12. The graph shows a $20-30 \%$ increase in the number of packets received by destination nodes as compared to data reception of our original model. This increase is due to the fact that a link between two nodes may be maintained when one node jumps to the next doorway, as opposed to entering the building. However, the packet delivery is still not as high as the random waypoint model because the movement from one doorway to another can result in an unrepairable link break.

Based on our results we hypothesize that two mechanisms could be utilized to improve data delivery. The first is to permit communication between nodes interior and exterior to obstacles based on the composition of the obstacle walls. The second is to have the source node periodically reattempt unsuccessful route discoveries throughout the simulation. That way, if a route later becomes available due to the movement of nodes, data packet delivery could resume. The investigation of both of these mechanisms is an area of future work.
The control overhead is shown in figure 13. The graph shows that the number of control packets transmitted by the obstacle model is significantly lower than in the random waypoint. This result is directly correlated with the number of failed data sessions. Because many data sessions are aborted, fewer sessions are maintained throughout the simulation, resulting in less overhead.

Figure 14 shows the end-to-end data packet delivery delay. This measurement includes route acquisition latencies for discovering the route. The figure shows that the data delivery delay for the obstacle model is significantly lower than in the random waypoint model. Because there are fewer data sessions that are able to be completed, there is less data traffic in the network overall. Hence data packets experience less contention for transmission and are able to be delivered more quickly to their destinations.

\section{CONCLUSIONS}

This paper describes a new mobility model that enables the inclusion of obstacles in ad hoc network simulations. The user specifies the placement of polygons within the simulation area by defining the coordinates of the obstacles. The model then incorporates the obstacles into the simulation terrain and calculates pathways between the obstacles using the Voronoi path computation. The obstacles are used both to define the movement pathways of the mobile nodes, and to obstruct the transmission of the nodes. Each time a node transmits a packet, the model determines whether the intended recipient of the packet is within the obstruction set of the transmitting node. If so, reception of the packet is blocked.

The simulation results of the obstacle model and the random waypoint model concur with a previous mobility model comparison [6] in that the mobility model significantly impacts the performance of an ad hoc network routing protocol. Through the use of the AODV protocol, we have shown that the mobility model effects a variety of characteristics, including the connectivity of the nodes and network density, as well as the packet delivery and overhead of the routing protocol. 
There are a number of ways to extend this initial work. The first of these relates to the selection of destinations. In this model each destination site has a nonzero probability of being selected by a given node. In reality, it is often the case that people travel most frequently between buildings located physically close to each other and travel less frequently to buildings further away. This would be the case, for instance, in a college campus that had the science buildings clustered in one area and the humanities buildings in another area. Upper-division science students would be likely to move within the science area and would travel less frequently into the humanities area. The opposite would be true for the humanities students. To model this phenomenon, destination selection could be exponential based on the distance between the potential destination and the node's current position.

In addition to using an exponential distribution for destination selection, it is also sometimes that case that certain locations act as attraction points for people at specific times. For instance, the university center is likely to be a popular destination during the lunch hour if a variety of meal options are available. Concerts, lectures, and special events on a campus can all act as attraction points where individuals from all areas of the campus flow to one area at a specified time. We would like to investigate the modeling of such attraction points and the impact on throughput and network performance resulting from such concentrated traffic areas.

In the current model, the intersecting sites are determined by the Voronoi computation, as described in section 4.2. This can result in occurrences of non-optimal doorway placement, for instance where two doorways are located on the same side of an object. To enable realistic doorway placement, or to exactly model existing buildings, the user should be allowed to indicate the placement of the doorways on the object sides. These preferences can then be considered in the Voronoi computation, such that the graph is modified to include these new points.

Finally, this work can also be improved through higher granularity modeling of transmissions through and around buildings. In this initial model, we have assumed that buildings are opaque and completely block signal propagation. In reality, many buildings do permit the propagation of wireless signals through their exterior walls. The quality of the signal penetration is a function of the composition and the thickness of the wall. We would like to develop a method for modeling this characteristic of the objects. Non-blocking walls would be likely to have a significant impact on the network topology characteristics. For instance, the average node density and data packet delivery would be higher if nodes interior to buildings were not isolated from the rest of the network.

The specific values obtained in our simulations are strongly dependent on the configuration of the obstacles in the network terrain. However, the data leads to an important conclusion. The results show that a wide range of scenarios must be studied to discern the overall performance of the routing protocol. Testing of a broader range of network conditions and topologies is needed for a complete understanding of ad hoc routing protocol performance. We intend to distribute our model as a plug-in to the GlomoSim simulator so that it can be useful to other wireless network researchers.

\section{REFERENCES}

[1] L. Bajaj, M. Takai, R. Ahuja, K. Tang, R. Bagrodia, and M. Gerla. GlomoSim: A Scalable Network Simulation Environment. Computer Science Department Technical Report, \#990027, UCLA, 1997.

[2] C. Bettstetter. Smooth is Better than Sharp: A random Mobility Model for Simulation of Wireless Networks. In Proceedings of the $4^{\text {th }} A C M$ International Workshop on Modeling, Analysis, and Simulation of Wireless and Mobile Systems (MSWIM), Rome, Italy, July 2001.

[3] C. Bettstetter, H. Hartenstein, and X. Perez-Costa. Stochastic Properties of the Random Waypoint Mobility Model: Epoch Length, Direction Distribution and Cell Change Rate. In Proceedings of the $5^{\text {th }} A C M$ International Workshop on Modeling, Analysis, and Simulation of Wireless and Mobile Systems (MSWIM), Atlanta, GA, September 2002.

[4] C. Bettstetter and C. Wagner. The Spatial Node Distribution of the Random Waypoint Mobility Model. In Proceedings of the $1^{\text {st }}$ German Workshop on Mobile Ad hoc Networks (WMAN), pages 41-58, Ulm, Germany, March 2002.

[5] J. Broch, D. A. Maltz, D. Johnson, Y.-C. Hu, and J. Jetcheva. A Performance Comparison of Multi-Hop Wireless Ad Hoc Network Routing Protocols. In Proceedings of the $4^{\text {th }}$ Annual ACM/IEEE International Conference on Mobile Computing and Networking (MobiCom), pages 85-97, Dallas, Texas, October 1998.

[6] T. Camp, J. Boleng, and V. Davies. A Survey of Mobility Models for Ad Hoc Network Research. Wireless Communications $\&$ Mobile Computing (WCMC): Special issue on Mobile Ad Hoc Networking: Research, Trends and Applications, 2(5):483-502, 2002.

[7] V. Davies. Evaluating Mobility Models Within an Ad hoc Network. Master's thesis, Colorado School of Mines, 2000.

[8] E. W. Dijkstra. A Note on Two Problems in Connection with Graphs. Num. Mathematik, 1:269-271, 1959.

[9] R. A. Guerin. Channel Occupancy Time Distribution in a Cellular Radio System. IEEE Transactions on Vehicular Technology, 36(3):89-99, 1987.

[10] Z. Haas. A New Routing Protocol for Reconfigurable Wireless Networks. In Proceedings of the IEEE International Conference on Universal Personal Communications (ICUPC), pages 562-565, October 1997.

[11] Z. J. Haas and M. R. Pearlman. The Performance of Query Control Schemes for the Zone Routing Protocol. In Proceedings of SIGCOMM, pages 167-177, Vancouver, British Columbia, 1998.

[12] X. Hong, M. Gerla, G. Pei, and C.-C. Chiang. A Group Mobility Model for Ad hoc Wireless Networks. In Proceedings of the ACM/IEEE MSWIM'99, Seattle, WA, August 1999. 
[13] S.-J. Lee, E. M. Royer, and C. E. Perkins. Ad hoc Routing Protocol Scalability. ACM/Wiley International Journal of Network Management, 13(2):97-114, March 2003.

[14] B. Liang and Z. Haas. Predictive Distance-based Mobility Management for PCS Networks. In Proceedings of the IEEE Conference on Computer Communication (INFOCOM), New York, NY, March 1999.

[15] M. de Berg, M. van Kreveld, M. Overmars and O. Schwarzkopf. Computational Geometry: Algorithms and Applications. Springer Verlag, 2000.

[16] M. R. Pearlman, Z. J. Haas, P. Sholander, and S. S. Tabrizi. On the Impact of Alternate Path Routing for Load Balancing in Mobile Ad hoc Networks. In Proceedings of the $1^{\text {st }}$ Annual Workshop on Mobile and Ad hoc Networking and Computer (MobiHOC)), pages 3-10, Boston, MA, August 2000.

[17] C. E. Perkins and E. M. Royer. The Ad hoc On-Demand Distance Vector Protocol. In C. E. Perkins, editor, Ad hoc Networking, pages 173-219. Addison-Wesley, 2000.

[18] G. Resta and P. Santi. An Analysis of the Node Spatial Distribution of the Random Waypoint Mobility Model for Ad hoc Networks. In Proceedings of the $2^{\text {nd }}$ ACM International Workshop on Principles of Mobile Comptuing (POMC), Toulouse, France, October 2002.

[19] E. M. Royer, P. M. Melliar-Smith, and L. E. Moser. An Analysis of the Optimum Node Density for Ad hoc Mobile Networks. In Proceedings of the IEEE International Conference on Communications, pages 857-861, Helsinki, Finland, June 2001. 